JOURNAL DE PHYSIQUE

ColZoque C4, supplément au $n^{\circ} 12$, Tome 43, décembre 1982

page $\mathrm{C4}-185$

\title{
TRANSMISSION ELECTRON MICROSCOPY OF MARTENSITIC INTERFACES
}

\author{
K.M. Knowles*, J.W. Christian and D.A. Smith** \\ University of Oxford, Department of Metallurgy and Science of Materials, \\ Parks Road, Oxford, England
}

(Accepted 9 August 1982)

\begin{abstract}
By observing suitable systems in the transmission electron microscope (t.e.m.), it is possible to obtain useful information on the structure of parent-martensite, transformation twin and intervariant interface. In this article, we review our recent work on $\mathrm{Ni}-\mathrm{Ti}$, $\mathrm{Ti}-\mathrm{Mn}$ and $\mathrm{Au}-\mathrm{Cd}$ alloys using both conventional and high resolution t.e.m., and we show how these techniques can be used to increase our understanding of martensitic interfaces.
\end{abstract}

\begin{abstract}
Introduction.- Transmission electron microscopy (t.e.m.) is now an established method for characterising martensitic materials (e.g. (1)). It is frequently used to obtain j.nformation on habit planes, parent-martensite orientation relationships and transformation twinning modes and the results are compared with the predictions of the formal crystallographic theories of martensite (2-4). In recent years, improvements in microscope reslutions have allowed the use of the high resolution t.e.m. technique of lattice fringe imaging to observe the fine structure of materials at a near-atomic leve1. In the case of martensitic materials, this can, in favourable circumstances, nermit the detailed examination of parent-martensite, transformation $t$ win and intervariant interfaces. The migration of such interfaces is an essential part of martensitic transformations and can also play a major part in the deformation characteristics of martensitic materials (5). In this article, we review our recent work on three non-ferrous martensitic systems and show how both convential and high-resolution t.e.m. have enhanced our understanding of martensitic interfaces in these systems.
\end{abstract}

\section{Electron Microscope Observations}

2.I Titanium - 5 wt $\%$ manganese.- In this alloy, the parent b.c.c. $\beta$ phase transforms martensitically on quenching to produce the low temperature h.c.p. $\alpha$ phase. In addition, the $\beta$ phase can transform to an h.c.p. $\omega$ phase during the quench. This system was originally investigated by Hammond and Kelly (6) who observed that the $\alpha$ martensite appeared in two morphologies, one as broad bands with a planar parentmartensite interface and one internally twinned with a zig-zag parent-martensite interface. Hammond and Kelly interpreted the two morphologies in terms of the two different solutions of the crystallographic theory for the b.c.c. - h.c.p. transformation. In order to explain the zig-zag interfaces, they had to postulate the presence of small local interface dilatations at each parent-twin interface. This approach al so requires steps in the zig-zag interfaces which, if large enough, might be detectable in the t.e.m.

* Now at the Department of Metallurgy and Materials Technology, University of Surrey, Guildford GU2 $5 \mathrm{XH}$, England

**Now at the Thomas J.Watson Research Center, IBM Corporation, Yorktown Heights, N.Y. 10598, U.S.A. 
We have recently re-examined this alloy system (7) and have shown that the two particular morphologies observed are in fact related and correspond to the same solution of the phenomenological theory as far as the orientation relationships are concerned. An example where the broad band morphology changes into the zig-zag morphology is shown in fig.1. Trace analyses of the parent-martensite interfaces were consistent with those quoted by Hammond and Kelly, but neither conventional two-beam dark field electron microscopy nor weak-beam imaging detected any interfacial structure. An alternative explanation for the observed interface orientation was formul ated using the surface dislocation approach (6) and without the need to invoke a dilatation parameter. The model suggests that the occurrence of interface faceting in materials such as $\mathrm{Ti}-\mathrm{Mn}$ is a consequence of the individual parent-twin interfaces lowering their net Burgers vector contents. One consequence of this approach is that the formal surface dislocations accommodating the misfit in the parent-martensite interfaces are not glissile, in contrast to the usual models, and one would expect that the motion of such interfaces would be impeded.

In a further investigation of $\mathrm{Ti}-5$ wt $\% \mathrm{Mn}$, a high resolution t.e.m. was used in the axial illumination modie: to obtain, where possible, two dimensional lattice fringe images from the parent and martensite (8). Fig.2 shows a lattice image of the parent martensite interface from a plate with the broad band morphology; the zone axes are $[001]_{\alpha} \|[1 \overline{10}]_{\beta}$ and the interface is parallel to the electron beam within experimental error. Computer simulations of the image demonstrate that the hexagonal array of bright dots characterising the $\alpha$ phase correspond to the "holes" in the $[001]_{\alpha}$ projected potential. The fringes characterising the $\beta$ phase correspond to either the (110) atomic planes or to the channels between the (110) planes, the position of the fringes being a sensitive function of defocus. This image cannot therefore yield atomistic information on the structure of the parent-martensite interface, although it is apparent that there are no large scale distortions in the interface region. Further aspects of the interpretation of lattice images from titanium-manganese are considered in reference (8), which al so gives examples of images from the transformation twin interfaces.

2.2 Equiatomic nickel-titanium. - The recent strong interest in shape memory arose most directly from observation of the effect in nickel-titanium alloys first reported by Buehler et al (9). Since this first report, there have been numerous investigations on the rather complex microstructure of the alloys. The first transformation twinning mode observed in the monoclinic $\mathrm{Ni}-\mathrm{Ti}$ martensite wa s a Type $\mathrm{I}$ twin with the twin plane $\mathrm{K}_{1}=(11 \overline{1})$, taking the convention that the obtuse angle in the monoclinic unit cell is the angle $\beta$ (10). Subsequently, Gupta and John son reported a Type I twinning mode wh $\mathrm{K}_{1}=(001)$ and al so reported details of samples in which they concluded that the martensite was composed of variants of the same monoclinic structure bearing no fixed orientation relationship to one another, but with a common (III) interface plane and an alternating platelet morphology (11).

Our first study on equiatonic nickel-titanium had two objectives (12): to clarify the observations of Gupta and Johnson and to apply the crystallographic theory to the otserved twinning modes. New experimental evidence showed that the alternating platelet morphology observed by Gupta and Johnson represents internal twinning by a Type II twin mode with the twin plane (0.72054 11). Application of the phenonenological theory to the various twinning modes gave predictions which were in good agreement with the available experimental results for the Type $I$ (11) and the Type II mode. The third twinning mode, Type $I$ with $\mathrm{K}_{1}=(001)$ is unable to produce the lattice invariant shear and is therefore more likely to be a deformation twinning rather than a transformation twinning mode. 


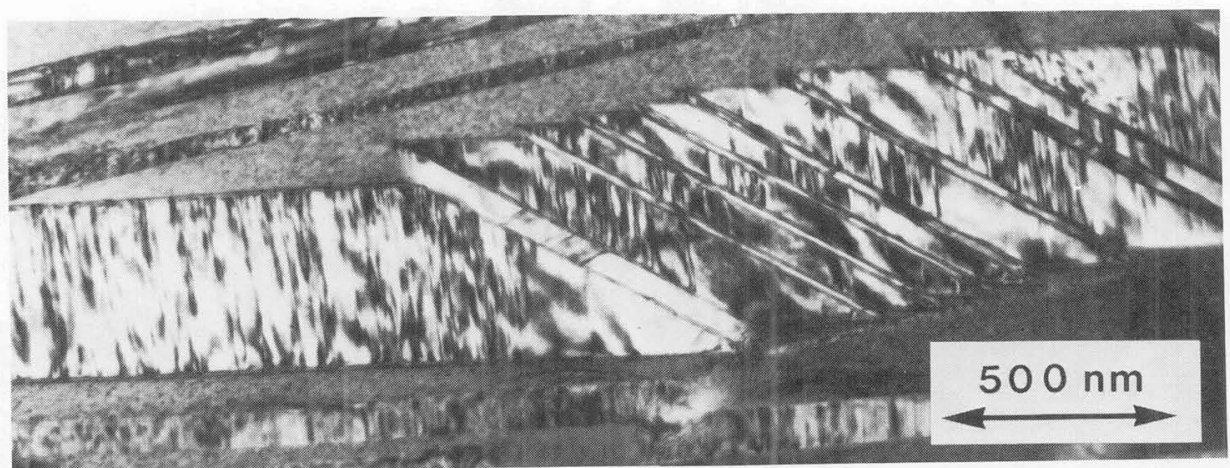

Fig.1 : Broad band morphology in Ti-Mn changing into transformation twinning zigzag morphology.

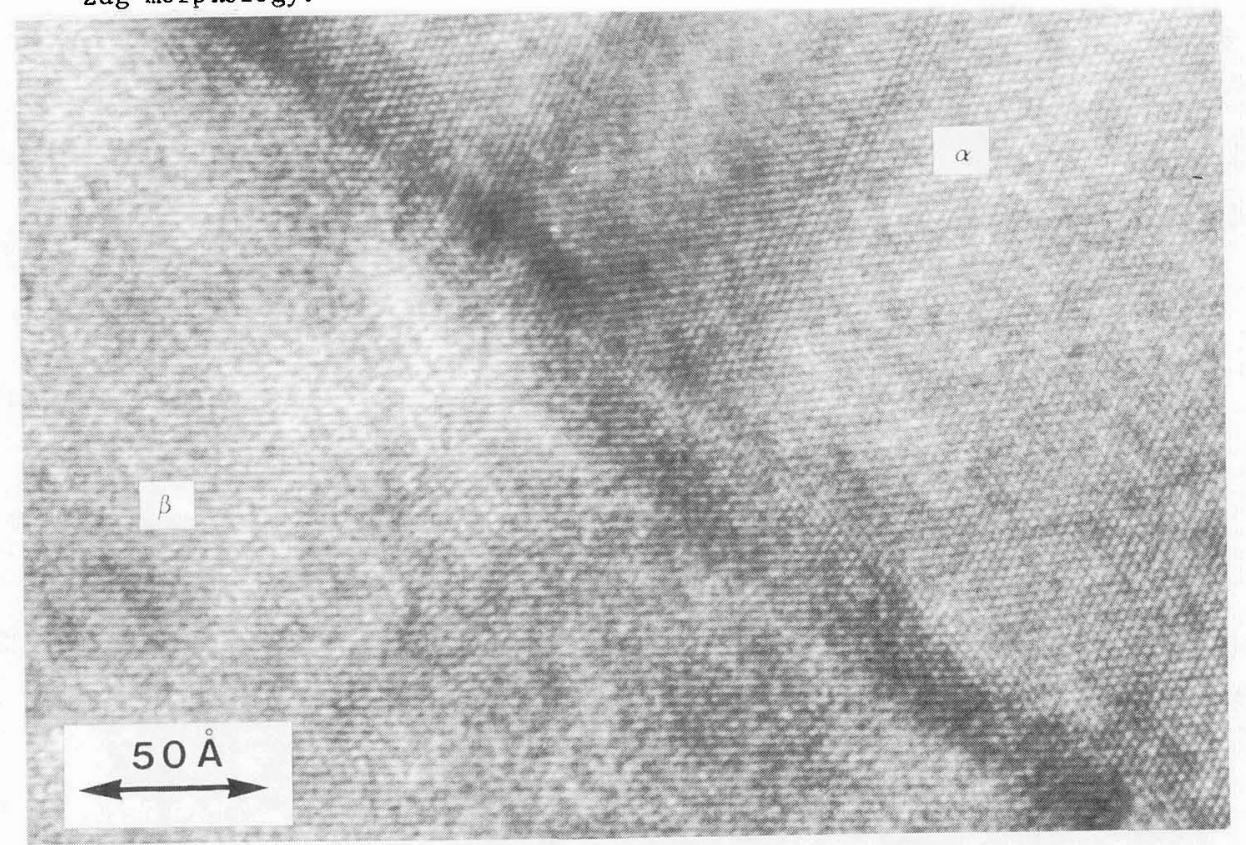

Fig.2 : Lattice fringe image of a parent martensite interface in Ti-Mn. The interface runs diagonally across the picture from top left to botton right and is almost parallel to the electron beam. Zone axes $[001]_{\alpha} \|[i \overline{10}]_{\beta} ; \Delta \mathrm{F}=-500 \AA$

Further investigations $(13,14)$ on this alloy system have used high resolution t.e.m. to examine the nature of the transformation twin, deformation twin and inter variant interfaces, all of which have to be relatively mobile (5). This work has, for the first time, show evidence for the step and ledge structure of Type II twin interfaces (13), with the nearest low index rational plane forming the ledges, as predicted theoretically in models of such interfaces. Observations on small areas with (001) twinning showed features consistent with twinning dislocations, which are steps with dislocation character, at the twin interfaces and al so faulting on (001) planes within the twins. Occasionally, twin terminations were observed (fig.3), which can be explained by having twinning dislocations accomplishing the tapering of the twin. High resolution t.e.m. of the intervariant interfaces was difficult to interpret quantitatively (14) because of the complex geometry associated with such interfaces 


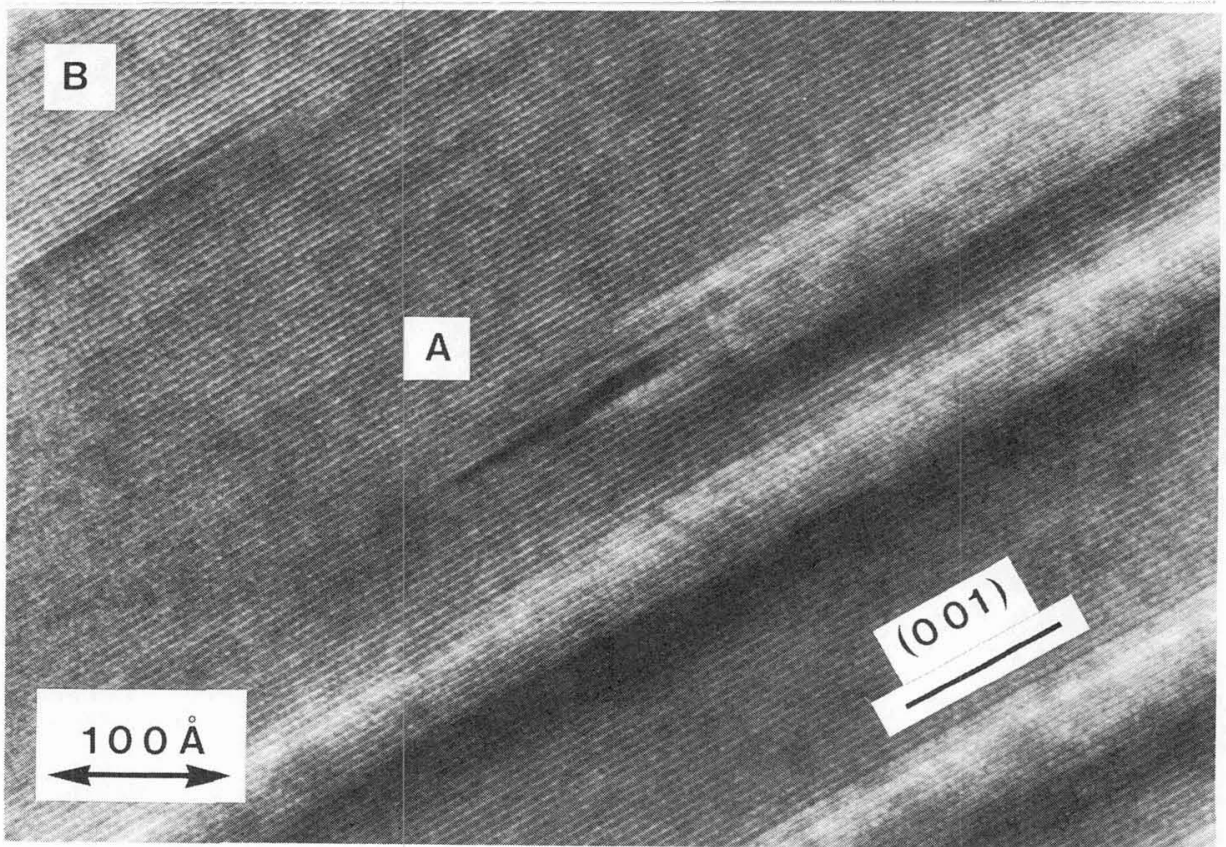

Fig.3 : Lattice fringe image of a terminating twin in $\mathrm{Ni}-\mathrm{Ti}$ (A) showing the illdefined nature of the termination, from (001) twins looking down the [1 $\overline{10}]$ zone. Faulting on $(001)$ can be seen at $\mathrm{B} . \Delta \mathrm{F}=-700 \AA$

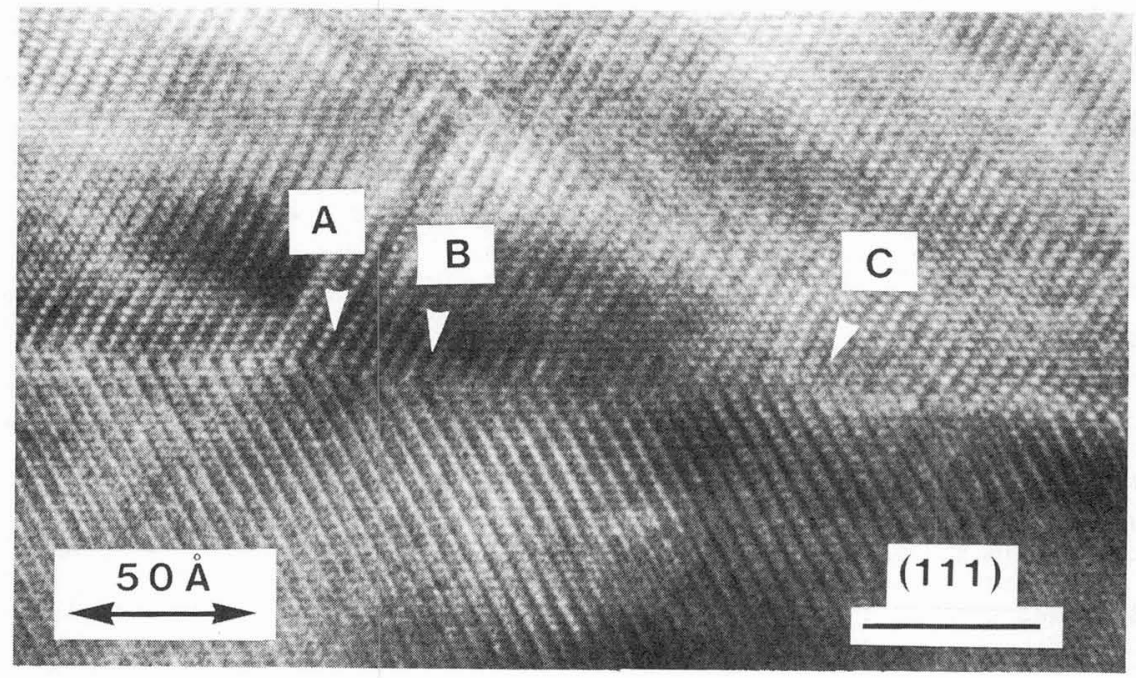

Fig.4 : A two dimensional lattice fringe image of a twin interface in Au-Cd showing steps at $A, B$ and $C . \Delta F=-500 A$ 
in most cases. Nevertheless, it is clear that further such investigations are necessary to understand fully the phenomenon of variant-variant coalescence at a microscopic level.

2.3 Gold -47.5 at \% cadmium.- The martensitic phase of Au-47.5 at \% Cd exhibits a remarkable rubber-like elasticity which has been attributed to the reversible motion of twin boundaries in the martensite (15). These twin boundary movements can be accomplished by glide of twinning dislocations. It might be expected that such twinning dislocations would be readily observable using the lattice imaging techninue, and this prompted a short investigation of a fully martensitic Au-47.5 at \% Cd alloy (16).

Fig. 4 is a portion of a twin interface with the diffraction conditions shown in fig.5. It is apparent from fig. 4 that the twin interface is not accurately parallel to (111), the $K_{1}$ plane of the transformation twinning mode in this alloy. The deviation a way from (111) is accomplished by three steps, two of height $2 \mathrm{~d}_{111}$ perpendicular to the (111) plane at $A$ and $B$ and one of height $3 d_{11}$ perpendicular to the

Fig.5 : Diffraction pattern used to form the lattice image in fig.4.Zone axes [1]̄n] in both twins, with the twin plane (111)

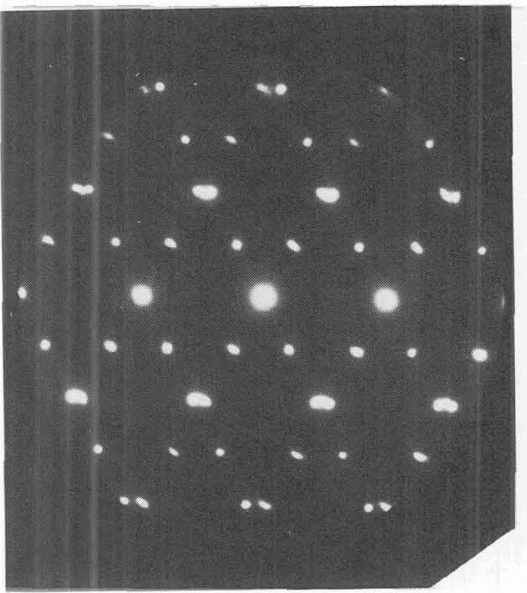

(111) plane at $C$, where $d_{111}=2.310 \AA$ is the interplanar spacing of the (111) planes. In all cases the steps appear to be parallel to the electron beam within experimental error and to lie on (001) planes of the lower twin. Particularly noteworthy is the fact that the steps do not all have the same height and that in all cases they are multiples of the unit step height dlll which one might expect from a twinning dislocation. Further analysis is not possible because the images could not be interpreted directly in terms of the projected potential distributions of the twins along the electron beam direction.

3. Concluding Remarks.- From the preceding examples, it is clear that both conventional and high resolution t.e.m. can provide valuable information on the structure of martensitic interfaces which one cannot expect to obtain by any other technique. In particular, we hope that the high resolution t.e.m. work presented here will stimulate further interest in the use of this technique for observations of the fine structure of interfaces. However, we recognise that current microscope resolution capabilities are insufficiently good to be able to differentiate unambiguously between various specific atomistic models of interfaces obtained from computer simulations. Nevertheless, with the resolution of electron microscopes improving all the time (Cambridge University, for example, have just modified the pole-pieces on their $575 \mathrm{kV}$ HREM and anticipate a possible instrumental resolution of $1.5 \&$ (17), such a differentiation may be possible fairly soon.

Acknowledgements. - We would like to thank Professor Sir Peter Hirsch F.R.S. for the provision of laboratory facilities and the Science Research Council for financial support throughout the course of this work. We would also like to thank friends and colleagues at Oxford for useful discussions on aspects of this work, particularly on the interpretation of the high resolution t.e.m. images. 


\section{References}

1. Wayman, C.M., in 'Diffraction and Imaging Techniques in Material Science', ed. S. Arnelinckx, R. Grevers and J. Van Landuyt, (North Ho1land : Amsterdam), vol.1, p.251 (2nd ed : 1978).

2. Wechsler, M.S., Lieberman, D.S. and Read, T.A., Trans. A.I.M.E. 197, 1503 (1953).

3. Bowles, J.S. and Mackenzie, J.K., Acta Met.,ㄹ, 129, 138, 224 (1954),

4. Bullough, R. and Bilby, B.A., Proc. Phys. Soc., B69, 1276 (1956).

5. Christian, J.W., Met. Trans. A, 13, 509 (1982).

6. Hammond, C. and Kelly, P.M., Acta Met., 17, 869 (1969).

7. Knowles, K.M. and Smith, D.A., Acta Met.,29, 1445 (1981).

8. Knowles, K.M., Proc. Roy. Soc. Lond. A, 380, 187 (1982).

9. Buehler, W.J., Gilfrich, J.v. and Wiley, R.C., J. App1. Phys., 34, 1475 (1963).

10. Otsuka, K., Sawamura, T. and Shimizu, K., Phys. stat. sol., 5, 457 (I971).

11. Gupta, S.P. and Jobnson, A.A., Trans. J.I.M., 14, 292 (1973).

12. Knowles, K.M. and Smith, D.A., Acta.Met., 29, 101 (1981).

13. Knowles, K.M., Phi 1. Mag., 45, 357 (1982).

14. Knowles, K.M. and Christian, J.W., Proceedings of the International Conference on Solid-Solid Phase Transformations, Pittsbürgh (1981), in the press.

15. Lieberman, D.S., Schmerling, M.A. and Karz, R.W., in 'Shape Memory Effects in Alloys', ed. J. Perkins (Plenum Press : New York), p.203 (1975).

16. Knowles, K.M., Inst. Phys. Conf. Ser. No.61, p.413 (1981).

1.7. Smith, D.J., private communication. 